\title{
Prediction and Simulation Using Simple Models Characterized by Nonstationarity and Seasonality*
}

\author{
Norman R. Swanson ${ }^{1,2}$ and Richard Urbach ${ }^{2}$ \\ ${ }^{1}$ Rutgers University \\ ${ }^{2}$ Conning Germany GmbH
}

revised August 21, 2013

\begin{abstract}
In this paper, we provide new evidence on the empirical usefulness of various simple seasonal models, and underscore the importance of carefully designing criteria by which one judges alternative models. In particular, we underscore the importance of both choice of forecast or simulation horizon and choice between minimizing point or distribution based loss measures. Our empirical analysis centers around the implementation of a series of simulation and prediction experiments, as well as a discussion of the stochastic properties of seasonal unit root models. Our prediction experiments are based on analysis of a group of 14 variables have been chosen to closely mimic the set of indicators used by the Federal Reserve to help in setting U.S. monetary policy, and our simulation experiments are based on a comparison of simulated and historical distributions of said variables using the testing approach of Corradi and Swanson (2007a). A key impetus for this paper stems from the fact that various financial service companies routinely create "economic scenarios", whereby seasonal and nonstationary financial and economic variables such as those examined here are simulated (and predicted) using relatively simple time series models. These "economic scenarios" are subsequently used in risk management and asset allocation, as is often mandated by various world financial regulatory authorities. Our findings suggest that a simple version of the seasonal unit root (SUROOT) model performs very well for predicting 8 of 14 variables, when the forecast horizon is 1-step ahead. However, for horizons of greater than one-step ahead, our SUROOT model perform poorly when used for prediction, suggesting that parameter estimation error is crucial to understanding the empirical performance of such models. This "parameter estimation error" result is confirmed via a series of Monte Carlo experiments. Simulation experiments yield similar conclusions, although SUROOT models in this case are useful for constructing "forward" conditional distributions at 1- and 3-step ahead horizons. Interestingly, simple periodic autoregressions do not have this property, and are found to perform very well in both prediction and simulation experiments, at all horizons up to 60 months ahead.
\end{abstract}

JEL classification: C13, C22, C52, C53.

Keywords: seasonal unit root, periodic autoregression, difference stationary, prediction, simulation.

* Norman R. Swanson (nswanson@econ.rutgers.edu): Department of Economics, Rutgers University, 75 Hamilton Street, New Brunswick, NJ 08901, USA and Conning Germany GmbH, Norbert Strasse 29, 50670 Cologne, Germany. Richard Urbach (Richard.Urbach@Conning.com): Conning Germany GmbH, Norbert Strasse 29, 50670 Cologne, Germany. We are grateful to the guest editors of the special issue of this journal on "Advances in Financial Risk Management and Economic Policy Uncertainty," Shawkat Hammoudeh and Michael McAleer, to Hamid Beladi (the editor), and to an anonymous referee for useful comments on earlier drafts of this paper. We are also grateful to John Chao, Valentina Corradi, Eric Ghysels and Hashem Pesaran for useful comments and discussions. The authors have also benefited from the excellent research assistance provided by Geetesh Bhardwaj. 


\section{Introduction}

One of the most important characteristics of econometric models is that they are generally viewed as approximations to some unknown underlying data generating process. Furthermore, the extent to which these approximations are adequate is often assessed by evaluation of their prediction and simulation properties. ${ }^{1}$ Careful examination of these properties is important, given that various financial service companies routinely create "economic scenarios", whereby seasonal and nonstationary financial and economic variables such as those examined here are simulated (and predicted) using relatively simple time series models. These "economic scenarios" are subsequently used in risk management and asset allocation, as is often mandated by various world financial regulatory authorities. For example, insurance companies and banks are often interested in simulating future economic scenarios in order to evaluate measures of capital adequacy, possibly under a variety of alternative economic "starting conditions", product pricing rules, and regulatory environments. Governments, on the other hand, often utilize econometric forecasting models in order to predict economic variables of interest, such as interest rates and inflation, both of which play a crucial role in policy analysis. Our objective in this paper is to assess the adequacy of a number of simple seasonal models that can be used in the context of seasonal and nonstationary variables, and all of which should be viewed as approximations, in terms of prediction and simulation.

Seasonal models in economics have received considerable renewed attention since the seminal paper of Hylleberg, Engle, Granger and Yoo (HEGY: 1990) on seasonal unit roots. From an empirical perspective, many papers have documented the prevalence of seasonal unit roots in the data, and evaluated the predictive performance of various seasonal models, including seasonal unit root models, deterministic seasonal models, and periodic autoregressions. From a theoretical perspective, many papers have developed new testing methods and suggested various nonlinear seasonal models. A very few important contributions in this area include: Hylleberg $(1992,1994)$, Beaulieu and Miron (1993), Franses (1996a,b), Miron (1996), Miron and Beaulieu (1996), Franses, Hoek and Paap (1997), Franses and Vogelsang (1998), Osborn, Heravi and Birchenhall (1999), Ghysels and Osborn (2001), Franses and Paap (2002), Osborn (2002), Osborn and Rodrigues (2002), Franses and van Dijk (2005), and the references cited therein.

\footnotetext{
${ }^{1}$ Note that another natural "metric" by which the adequcy of econometric models can be evaluated is via examination of the properties of estimators used when analyzing and implementing the models.
} 
In this paper, we add to the above literature by revisiting some of the very simplest and more useful seasonal models, and evaluating their empirical forecasting and simulation properties. In particular, we use four econometric models to examine fourteen different economic variables measuring seasonally unadjusted economic activity. The variables considered are chosen becasue they are key economic and financial indicators used by the U.S. Federal Reserve to aid in the formulation of the nation's monetary policy (see below for further discussion). These variables examine various measures of industrial production, money, housing starts, employment, inventories, and sales; all of which are characterized by distinctive seasonal fluctuations. The models that we consider are: the seasonal unit root (SUROOT) model, a deterministic seasonality (DS) model, a periodic autoregression (PAR) model, and a strawman random walk (RW) model, possibly with drift. The choice of these models is predicated on two facts. First, these models nest (when allowing for ARMA type errors) a large class of models frequently used by industry specialists, when simulating seasonal economic variables. Second, these models are consistent with the oft touted empirical stylized fact that parsimonious models perform very well, when compared with more complicated and heavily parameterized models, in the context of forecasting a number of economic variables. Our analysis is based on the results of a series of Monte Carlo experiments, prediction experiments, and simulation experiments. Our Monte Carlo experiments evaluate SUROOT models and SUROOT tests, with emphasis on the effects of parameter estimation as forecast horizon increases. Our prediction experiments construct sequences of recursive ex -ante predictions using the alternative models, and assess mean square forecast error (MSFE) using point estimates as well as predictive accuracy tests. Finally, in a series of simulation experiments, we evaluate the distributional characteristics of data simulated using the four different model types. This is done by reviewing, and then implementing novel testing methodology developed by Corradi and Swanson (2007a), where empirical distributions of historical and simulated datasets are compared, using Kolmogorov type distributional tests (see also e.g., Andrews (1997)) based on distributional mean square error loss.

Our findings can be summarized as follows. We provide evidence that a simple version of the SUROOT model performs very well for predicting various macroeconomic variables when the forecast horizon is 1-step ahead. However, for horizons of greater than one-step ahead, the SUROOT models perform poorly when used for prediction, suggesting that parameter estimation error is crucial to understanding the empirical performance of such models. This result is confirmed via 
a series of Monte Carlo experiments. Interestingly, simple periodic autoregressions do not have this property, and indeed perform very well in both prediction and simulation experiments, at all horizons considered in this paper. Deterministic seasonal models also perform reasonably well at all forecast horizons, and indeed dominate PAR models for a small subset of our variables, including price and industrial production sub-component variables. Finally, by comparing simulation and prediction based evidence, we underscore the importance of carefully designing on a case by case basis the criteria with which one judges alternative models. A key take-away from our analysis is that we underscore the importance of carefully designing criteria by which one judges alternative models. In particular, we underscore the importance of both choice of forecast or simulation horizon and choice between minimizing point or distribution based loss measures.

The rest of the paper is organized as follows. In Section 2, we outline our empirical methodology and elucidate some relevant analytical properties of SUROOT models. In Section 3, we describe the data used. Section 4 contains the results of our Monte Carlo experiments, and Section 5 summarizes our empirical findings. Concluding remarks and recommendations are given in Section 6.

\section{Empirical Methodology}

\subsection{Models, Estimation and Diagnostic Tests}

There are a plethora of models from whence the "best" model for a particular time series can be chosen. These include both linear and nonlinear models, parametric and nonparametric models, and stochastic and deterministic models, for example. However, there is substantial empirical evidence that parsimonious (linear) models often yield more accurate predictions than more complex (nonlinear) models.

In this paper, we examine a small group of widely used univariate models, and check whether any of these outperform SUROOT models when used for simulation and prediction. These models are not meant to be exhaustive of the rich variety of models currently available to applied practitioners, but are rather meant to be a simple illustrative set of models that are useful for calibrating our experiments. We conjecture that the conclusions that are made via analysis of our experimental findings are broadly applicable, however. Still, whether the evidence that we gather can indeed be generalized to the case of more complex linear and nonlinear models is left to future research.

Let $y_{t}=(1-L) \ln X_{t}=\Delta \ln X_{t}$ be a scalar growth rate of some economic variable of interest. 
In the sequel we shall consider models of the form:

I. $y_{t}=\theta_{0}+\varepsilon_{t}-$ Random Walk (RW) Model

II. $\phi(L) y_{t}=\sum_{s=1}^{S} \theta_{s} d_{s, t}+\varepsilon_{t}-$ Difference Stationary (DS) Model (with deterministic seasonal components)

III. $\phi(L) \triangle_{S} y_{t}=\left(1+\theta L^{s}\right) \varepsilon_{t}-$ Seasonal Unit Root (SUROOT) Model

IV. $y_{t}=\theta_{s}+\sum_{i=1}^{p} \theta_{i, s} y_{t-i}+\varepsilon_{t}-$ Periodic Autoregression (PAR) Model

In these models, $\triangle_{S}$ denotes the $S^{\text {th }}$ difference operator, where $S$ is the number of seasons presumed to be in the data (i.e., $\triangle_{S}$ is the seasonal difference operator); $\phi(L)=\left(1-\phi_{1} L-\ldots-\right.$ $\left.\phi_{p} L\right)$ is a standard lag polynomial of order $p$, expressed using the lag operator, $L$; and the $\theta_{s}$ denote seasonal intercepts, with associated and conformably defined dummy variables, $d_{s, t}$. For an exhaustive discussion of the seasonal models considered in this paper, see the books by Hylleberg (1992), Franses (1996a), Miron (1996), and Ghysels and Osborn (2001); and for an interesting discussion on the usefulness of seasonal adjustment, see Franses (2001).

Notice that the PAR model generalizes the DS model, as it allows the intercept and slope coefficients to vary according to the season. Notice also that both of these models generalize the RW model, which is used as a "strawman" model in our analysis. In all cases, two versions of the random walk are fitted, one with $\theta_{0}=0$ and one with $\theta_{0} \neq 0$, and the model that performs better is reported on. Finally, notice that the SUROOT model is the same as that specified by Bell (1987), and Bell shows that our DS and SUROOT models are equivalent when $\theta=1$ (he actually sets $\phi(L)=1$, but further shows that the result holds when the error follows a general ARMA process). This variety of SUROOT model has been seen to perform relatively well in various empirical contexts (see e.g. Bell (1987), Ghysels and Osborn (2001), and the references cited therein).

All models are estimated using least squares, and the lag polynomial order is estimated using the Schwarz Information Criterion (SIC). The case where $p=0$ was allowed for, and indeed picked in some instances. Consider the $\operatorname{PAR}(1)$ model, where $p=1$. This model can be estimated via least squares using the equation: $y_{t}=\sum_{s=1}^{S} \theta_{s} d_{s, t}+\sum_{s=1}^{S} \theta_{1, s} d_{s, t} y_{t-1}+\varepsilon_{t}$. Here, under error normality and given fixed starting values, the least squares estimator is the maximum likelihood estimator, and standard asymptotics obtain (see Franses and Paap (2002) and Franses and van Dijk (2005) for further details). Notice that the $\operatorname{PAR}(1)$ model has a unit root when $\theta_{1,1} \theta_{1,2} \theta_{1,3} \cdots \theta_{1, s}=1$. Clearly, the PAR(1) model nests the simple random walk model where $\triangle_{1} y_{t}=(1-\theta L) y_{t}=\theta_{0}+\varepsilon_{t}$, 
with $\theta=1$. In this case, the characteristic equation is $\left(1-\theta^{S} z\right)=0$, so that when $\theta=1$, $y_{t}$ has a single nonseasonal unit root, corresponding to the simple random walk model. Also, when $\theta=-1$, $y_{t}$ has a seasonal unit root. Thus, as mentioned above, both seasonal and non-seasonal unit root processes such as those given in our RW, DS, and SUROOT models, are nested within PAR models (see HEGY (1990) for further details). Nonlinear variants of the above models are discussed in Franses and van Dijk (2005), and Rodrigues (2001), and the papers cited therein. Boswijk and Franses (BF: 1996) outline tests for $\theta_{1, s} \theta_{2, s} \theta_{3, s} \theta_{4, s}=1$ and for $\theta=1$ and $\theta=-1$ (see Franses and Paap (2002) for a summary; and Granger and Teräsvirta (1993) for a summary of the types of nonlinear models used by these authors).

Diagnostic tests used in the sequel include Jarque-Bera, augmented Dickey-Fuller (ADF) (with lags selected via use of the SIC), BF periodic integration, and HEGY (called HEGY1) seasonal unit root tests. In addition, we use the HEGY2 test, which refers to a HEGY1 test with pre whitening, and involves estimating $\widehat{\triangle_{S}} y_{t}=\sum_{i=1}^{p} \widehat{\phi}_{i} \triangle_{S} y_{t-i}$. HEGY1 is then applied to pre-whitened data $y_{t}^{*}=\left(1-\sum_{i=1}^{p} \widehat{\phi}_{i} L^{i}\right) y_{t}$ as suggested by Psaradakis (1997). Note that in this paper, we report test results for the null of no seasonal unit root based on examination of $\pi_{2}$ (see Beaulieu and Miron (1993) for complete details, and Swanson and Urbach (2013) for complete HEGY test results at all frequencies, which are omitted here for the sake of brevity).

One of the most recognized features of SUROOT models, the notion that winter can become summer, is perhaps worth recalling before we turn to a discussion of the empirical methods used in the sequel. Consider the very simple model where $\triangle_{S} y_{t}=\theta_{0}+\varepsilon_{t}$, and where $y_{t}$ is driven by a nonstationary process in each season. Now, these seasons might share a common drift, $\theta_{0}$, but their evolution is clearly independent from one season to the next, given that the evolution of each season can be written as $y_{t}^{s}=\theta_{0}+\varepsilon_{s, t}$, where $\varepsilon_{s, t} \sim$ iid $N\left(0, \sigma^{2}\right)$, say, for $s=1, \ldots, S$. Thus, $\triangle_{S} y_{t}$ can be viewed as being comprised of $S$ independent random walks (see e.g. Osborn and Ghysels (2001) and Franses and van Dijk (2005)). This suggests that when used for simulation or prediction, one should expect values of $y_{t}$ in different seasons to drift infinitely far apart, given enough time (in the case of the simple SUROOT model examined in this paper). Clearly this is a feature which is not in accord with expected behavior of economic series, at least in the long run. However, all models should be viewed as approximations, and hence the above feature may be acceptable, as long as a particular SUROOT model still yields adequate predictions and simulated observations for a given time series, over horizons that the researcher is interested in. To illustrate 
the importance of this point, consider Figure 1, where inflation, measured as $y_{t}=\Delta \ln X_{t}$, with $X_{t}=C P I$, is simulated under the assumption that either (i) CPI follows a random walk with drift in logs, or (ii) $\triangle_{S} y_{t}=\theta_{0}+\varepsilon_{t}$. Here, models are estimated using monthly U.S. data for the period 1959-2005. Notice that $y_{t}$ data generated via the SUROOT simulation model are increasing over time, with ever increasing volatility, a feature which is clearly not in accord with the historical record, and which suggests that care needs to be taken when using this model over long horizons. ${ }^{2}$ This observation plays an important role in our subsequent findings.

\subsection{Simulation and Predictive Accuracy Testing Methodology}

We begin by summarizing the simulation based distributional accuracy test discussed in Corradi and Swanson (CS: 2007a) for comparing simulated data with historical data. Assume that our objective is to compare the joint distribution of the historical data with the joint distribution of a simulated series. Following CS, and for the sake of simplicity, we limit our attention in the section to the evaluation of the joint empirical distribution of (actual and model-based) current and previous period values, say $y_{t}^{*}=\left(\Delta \ln X_{t}, \Delta \ln X_{t-1}\right)$, of our variable of interest, $y_{t}=\Delta \ln X_{t}$. Consider $m$ alternative econometric models, and set model 1 as the benchmark model. Let $\Delta \ln X_{j, n}\left(\widehat{\theta}_{j, T}\right)$, $j=1, \ldots, m$ and $n=1, \ldots, N$, denote the series of interest, simulated under model $j$, where $N$ denotes the length of the simulated sample, and $\widehat{\theta}_{j, T}$ denotes estimated parameters, using the $T$ available historical observations. Thus, $y_{j, n}^{*}\left(\widehat{\theta}_{j, T}\right)=\left(\Delta \ln X_{j, n}\left(\widehat{\theta}_{j, T}\right), \Delta \ln X_{j, n-1}\left(\widehat{\theta}_{j, T}\right)\right)$. (In general, one can set $y_{t}^{*}$ to be any stationary vector of economic variables.) Also, let $F_{0}\left(u ; \theta_{0}\right)$ denote the distribution of $y_{t}^{*}$ evaluated at $u$ and $F_{j}\left(u ; \theta_{j}^{\dagger}\right)$ denote the distribution of $y_{j, n}^{*}\left(\theta_{j}^{\dagger}\right)$, where $\theta_{j}^{\dagger}$ is the probability limit of $\widehat{\theta}_{j, T}$, taken as $T \rightarrow \infty$, and where $u \in U \subset \Re^{2}$, possibly unbounded. Accuracy is measured in terms of square error. The squared (approximation) error associated with model $i$, $i=1, \ldots, m$, is measured in terms of the (weighted) average over $U$ of $E\left(\left(F_{i}\left(u ; \theta_{i}^{\dagger}\right)-F_{0}\left(u ; \theta_{0}\right)\right)^{2}\right)$. Thus, the rule is to choose Model 1 over Model 2 if:

$$
\int_{U} E\left(\left(F_{1}\left(u ; \theta_{1}^{\dagger}\right)-F_{0}\left(u ; \theta_{0}\right)\right)^{2}\right) \phi(u) d u<\int_{U} E\left(\left(F_{2}\left(u ; \theta_{2}^{\dagger}\right)-F_{0}\left(u ; \theta_{0}\right)\right)^{2}\right) \phi(u) d u,
$$

\footnotetext{
${ }^{2}$ Note that the two models ((i) and (ii) fit the data equally well, based on examination of in-sample correlation and residual serial autocorrelation. Moreover, thiese features can be tested for. However, in this paper we focus on simulation and prediction experiments, as we view these experiments as the more important metrics for model selection in our context, we do not carry out such tests.
} 
where $\int_{U} \phi(u) d u=1$ and $\phi(u) \geq 0$, for all $u$. The hypotheses of interest are:

$$
\begin{gathered}
H_{0}: \max _{j=2, \ldots, m} \int_{U} E\left(\left(F_{0}\left(u ; \theta_{0}\right)-F_{1}\left(u ; \theta_{1}^{\dagger}\right)\right)^{2}-\left(F_{0}(u)-F_{j}\left(u ; \theta_{j}^{\dagger}\right)\right)^{2}\right) \phi(u) d u \leq 0 \\
H_{A}: \max _{j=2, \ldots, m} \int_{U} E\left(\left(F_{0}\left(u ; \theta_{0}\right)-F_{1}\left(u ; \theta_{1}^{\dagger}\right)\right)^{2}-\left(F_{0}(u)-F_{j}\left(u ; \theta_{j}^{\dagger}\right)\right)^{2}\right) \phi(u) d u>0 .
\end{gathered}
$$

Under $H_{0}$, no model can provide a better approximation (in square error sense) to the distribution of $Y_{t}$ than the approximation provided by model 1. If interest focuses on confidence intervals, so that the objective is to "approximate" $\operatorname{Pr}\left(\underline{u} \leq y_{t} \leq \bar{u}\right)$, then the null and alternative hypotheses can be written in a form similar to that above, and appropriate statistics can be constructed. In order to test $H_{0}$ versus $H_{A}$, the relevant test statistic is $\sqrt{T} Z_{T, N}$, where $Z_{T, N}=\max _{j=2, \ldots, m} \int_{U} Z_{j, T, N}(u) \phi(u) d u$, and:

$Z_{j, T, N}(u)=\frac{1}{T} \sum_{t=1}^{T}\left(1\left\{y_{t}^{*} \leq u\right\}-\frac{1}{N} \sum_{n=1}^{N} 1\left\{y_{1, n}\left(\widehat{\theta}_{1, T}\right) \leq u\right\}\right)^{2}-\frac{1}{T} \sum_{t=1}^{T}\left(1\left\{y_{t}^{*} \leq u\right\}-\frac{1}{N} \sum_{n=1}^{N} 1\left\{y_{j, n}^{*}\left(\widehat{\theta}_{j, T}\right) \leq u\right\}\right)^{2}$

where $\widehat{\theta}_{j, T}$ is an estimator of $\theta_{j}^{\dagger}$. (The first term in the above expression is called the CS distributional loss for Model "1", while the second term is the loss for Model " $\jmath$ ".) Asymptotically valid critical values for this test can easily be constructed using the block bootstrap (see Corradi and Swanson (2007a) for further details). One crucial feature of the $Z_{T, N}$ test is that all models under both hypotheses may be misspecified, as opposed to the usual practice of assuming correct specification under the null.

We now turn to a discussion of pointwise predictive accuracy testing. For a discussion of predictive accuracy and predictive model selection tests available in the current literature, the reader is referred to Corradi and Swanson (2006a) and the references cited therein. In the current paper, we use the Diebold and Mariano (DM: 1995) and West (1996) test, where the equal predictive accuracy null is tested using:

$$
D M=\sqrt{P} \frac{\frac{1}{P} \sum_{t=R}^{T} \widehat{d}_{t+h}}{\frac{1}{P-h+1} \sum_{j=-\bar{j}}^{\bar{j}} \sum_{t=R+j}^{T-h} K\left(\frac{j}{M}\right)\left(\widehat{d}_{t+h}-\bar{d}\right)\left(\widehat{d}_{t+h-j}-\bar{d}\right)},
$$

where $\widehat{d}_{t+h}=\widehat{u}_{1, t+h}^{2}-\widehat{u}_{2, t+h}^{2}$ under mean square error loss; $\bar{d}=\frac{1}{P-h+1} \sum_{t=R}^{T-\tau} \widehat{d}_{t+h} ; \widehat{u}_{j, t+h}$ is an ex-ante forecast error constructed using a recursively estimated prediction model, for $j=1,2$; and $P$ is the number of observations in the ex ante forecast period. Of note is that loss functions other than mean square error loss can be used, although in this paper we focus exclusively on mean 
square forecast error loss. The limiting distributions of the DM statistic is given in Theorems 3.1 and 3.2 in Clark and McCracken (2005), and for $h>1$ contains nuisance parameters so that critical values cannot be directly tabulated, and hence Clark and McCracken (2005) use the Kilian (1999) parametric bootstrap to obtain critical values (see e.g. McCracken and Saap (2004) for discussion). Note, though, that for $h=1$, when models are nonnested (see Corradi and Swanson (2002) for a discussion of predictive accuracy and predictive Granger causality tests with nested models), and assuming that parameter uncertainty vanishes asymptotically, the standard normal distribution applies in the case of the DM test, even when the heteroskedasticity and autocorrelation consis-

tent standard error given in the numerator of $D M$ is replaced with $\frac{1}{P-h+1} \sum_{t=R+j}^{T-h}\left(\widehat{d}_{t+h}-\bar{d}\right)^{2}$. Furthermore, nonstandard critical values which obtain in other cases are generally larger, so that rejection of the null hypothesis using percentiles of the normal distribution often implies rejection using nonstandard critical values. Finally, nonstandard critical values are usually quite close, in absolute magnitude, to standard normal values. For these reasons, we use standard normal rejection regions when we report significance of DM test statistics. These should, of course, only be taken as a rough guide. For a thorough discussion of predictive accuracy tests, based both on point MSFEs and densities, the reader is referred to Corradi and Swanson (2006a,b). For a discussion of predictive accuracy testing under recursive estimation schemes such as that used here, the reader is referred to Corradi and Swanson (2007b). For a complete discussion of the properties of all of the diagnostic tests discussed in this section, refer to Corradi and Swanson (2006a, 2006b, 2007a, 2007b). For further discussion of distribution predictability, please see Zhu (2013); and for further discussion of forecasting economic and financial variables in general, see Cheng and Wu (2013).

\section{Data}

We examine 14 monthly U.S. series for the period 1959:1-2005:12, except where noted. The series include: two money stock variables (M1 and M2) and M3; two CPI series including CPI for all urban consumers, all items (CPI1), and energy (CPI2); housing starts (H_Start); industrial production $(I P)$; total nonfarm employment (NonF_Emp); industrial production - automotive products (IP_Auto); industrial production - durable consumer goods (IP_Dur); industrial production - durable consumer goods (IP_NDur); durable goods shipments of new orders and unfilled orders (D_Ship); total inventories, manufacturing (Invent); retail sales (Ret_Sales) - for the period 
1967:1-2001:4; and motor vehicle unit retail sales (Veh_Sales) - for the period 1967:1-2005:12. These variables have been extracted from the FRED (Federal Reserve Bank of St. Louis) database, the U.S. Census Bureau, and http://wwweconomagic.com. Plots of the series are presented in Figure 1 , where a variety of the series can be seen to exhibit cyclical variation consistent with seasonality.

Our group of 14 variables has been chosen to closely mimic a set of macroeconomic variables discussed by Federal Reserve Bank of New York on their website (from their list, we do not, however, examine yields and the S\&P500 index, both of which were found to exhibit little seasonality). The connection between our variables and macroeoconomic policy stems from the fact that observable economic variables are often used to proxy latent factors underlying the macroeconomic cycle, as espoused on the Federal Reserve Bank of New York's website, where it is was recently noted that: "In formulating the nation's monetary policy, the Federal Reserve considers a number of factors, including the economic and financial indicators which follow, as well as the anecdotal reports compiled in the Beige Book. Real Gross Domestic Product; Consumer Price Index; Nonfarm Payroll Employment Housing Starts; Industrial Production/Capacity Utilization; Retail Sales; Business Sales and Inventories; Advance Durable Goods Shipments, New Orders and Unfilled Orders; Lightweight Vehicle Sales; Yield on 10-year Treasury Bond; SEP 500 Stock Index; M2".

\section{Monte Carlo Results}

In this section, the results of a small Monte Carlo experiment carried out in order to assess the finite sample impact of parameter estimation error on SUROOT model predictive performance, and HEGY test size are reported. Data are generated according to the following SUROOT model:

$$
\phi(L) \triangle_{S} y_{t}=\left(1+\theta L^{s}\right) \varepsilon_{t}
$$

where $\phi(L)=1-\phi L$, and $\varepsilon_{t} \sim N\left(0, \sigma_{\varepsilon}^{2}\right)$. Parameter values are chosen to mimic estimates obtained from the 14 monthly series used in our empirical analysis. In particular, we set $\theta=$ $\{-0.6,-0.7,-0.8,-0.9\}, \phi=\{-0.5,0.0,0.5\}$, and $\sigma_{\varepsilon}=\{0.005,0.01,0.1\}$. Moreover, in order to mimic the data transformation used in our empirical analysis, we set $y_{t}=(1-L) \ln X_{t}$, and $s=12 .{ }^{3}$ Results based upon a (i) series of predictions experiments, and (ii) in-sample HEGY tests

\footnotetext{
${ }^{3}$ Results are reported in Table 2 for all cases except $\theta=\{-0.7,-0.8\}$, which are omitted for the sake of brevity, and because results for these cases can be immediately inferred from examination of results for the cases where $\theta=\{-0.6,-0.9\}$.
} 
are reported in the table. With regard to the prediction experiments, in each simulation, a sample of 500 observations was drawn, and prediction models were recursively estimated, beginning with an in-sample period of $120-h+1$ observations, and yielding a sequence of 380 ex-ante $h$-step ahead forecasts. Here, as elsewhere, $h$ is the forecast horizon (and is set equal to 1, 3, 12, and 60 months). ${ }^{4}$ Results reported in the first 12 columns of numerical entries in the Table 2 are based upon examination of these forecasts. Namely, the first 4 columns report the proportion of simulations for which the SUROOT model predictions were "MSFE-better" than those based on a Random Walk model (RW). The next 8 columns report the proportion of simulations for which the two models yielded "MSFE-better" predictions based upon application of the Diebold-Mariano (1995) predictive accuracy test at a $5 \%$ nominal significance level, and based on MSFE loss. Entries in the last three columns of the table indicate the proportion of times that the seasonal unit root (HEGY) and BF tests reject the null (see Table 1 and Section 2 for further details). All results are based on 500 Monte Carlo simulations.

A number of conclusions can be drawn from examination of the table. First, notice that in the point MSFE comparison, the SUROOT model is "MSFE-best" in every simulation for $h=1$ and $h=3$. However, for greater values of $h$, performance drops of, and indeed for $h=12$, when $\theta=-0.9$ the RW model actually "wins" around $50 \%$ of the time; and around $75 \%$ of the time when $h=60$. This suggests that parameter estimation error plays a substantive role when the forecast horizon is reasonably large, and when the MA coefficient is high, as may be expected to be the case in practice. Second, notice that the frequency of DM test "wins" agrees with the previous finding. Namely, for $h=12$ and $h=60$, the proportion of simulations for which the SUROOT model has a significantly lower MSFE than the RW model ranges from 25 to $20 \%$, even for cases where $\theta=-0.6$. Given that the true DGP is a SUROOT model, and the correct specification is nested within the class of estimated SUROOT models, it is quite clear that parameter estimation error truly plays a crucial role in our experiment. Indeed, for $h=60$ the random walk actually yields significantly lower MSFEs than the SUROOT model much more frequently than the number of times that SUROOT "wins", when $\theta=-0.9$. In some sense, this is not too surprising, given

\footnotetext{
${ }^{4}$ The choice of forecast horizons reported in our tabulated results is consistent with the duration of many prediction and simulation experiments carried out both in industry, with one key exception. In certain instances, such as in life insurance economic scenario simulations, 25 year simulations are often used, which are longer than our longest (5 year) simulations.
} 
that seasonal difference operators tend to cancel out with "near" non-invertible MA operators, as pointed out by Bell (1987), and observed in finite sample experiments by Ghysels, Lee and Noh (1994) and Rodrigues and Osborn (1999). Overall, this is an important finding that, when coupled with our discussion of Figure 1 (see above), underscores the importance of the choice of (prediction or simulation) horizon when carrying out model selection. Finally, notice that BF, HEGY1, and HEGY2 tests are very poorly sized in our experiment (see last three columns in the table). These results are consistent with earlier findings by many authors, including those just mentioned, and suggest that when using monthly data, standard seasonal unit root tests may be an inadequate guide as to the usefulness of such models. For discussion of testing procedures available that are designed to improve the finite sample properties of these tests, see e.g. Urga and P. Aroca-Gonzalez (2000), Franses (1996a,b), Ghysels and Osborn (2001), and the references on testing cited in the introduction to this paper. For this reason, it may be preferable in empirical applications to simply fit the different models and assess which models perform "best", based on simulation and prediction experiments, for example. Alternatively, carefully "adjusted" seasonality and nonstationarity tests might be developed and used.

\section{Empirical Results}

\subsection{Basic Data Analysis}

Given that BF and HEGY tests perform somewhat poorly using monthly data with time series properties of the type exhibited by the 14 economic variables analyzed in this section, we begin by searching for evidence of seasonality using a variety of means. First, estimated spectra are plotted in Figure 2. To interpret these plots, recall that a frequency of $\omega_{j}=\frac{2 \pi j}{T}$ corresponds to

a period of $\frac{2 \pi}{\omega_{j}}=\frac{T}{j}$ (see e.g., Hamilton (1994, chp. 6). In the plots, $j$ is reported on x-axis. For illustrative purposes, consider the time series D_Ship. The sample size is $T=563$. The first peak occurs around $j=93$, corresponds to a cycle of $563 / 93 \approx 6$ months. The second peak at $j=140$, corresponds to a cycle of 4 months, the third cycle is 3 months, and fourth cycle is around 2 months. Similarly for Ret_Sales, $T=419$. Thus, spectrum peaks at $j \in(33,68,102,136,170)$ correspond to cycles at around $12,6,4,3$ and 2 months. Evidently, the spectra suggest that seasonality is prevalent in a number of the series. However, note in Table 1 that HEGY and BF tests find little evidence of seasonality. Given the poor empirical size of these tests, however, this evidence 
should be interpreted with care. Indeed, when deterministic seasonal dummy models were fit to the data, many associated coefficients were found to be significantly different from zero based upon the application of $5 \%$ nominal size tests. The last column of the Table 1 supports this finding, as the null of no deterministic seasonality is rejected for all 14 variables (see Urbach and Swanson (2013) for complete tabulated HEGY test results and complete deterministic dummy variable estimation results). Overall, there is quite clear evidence of seasonality in our data. Furthermore, note that in Table 1, all series fail to reject the null of a unit root, based on application of the ADF test, supporting our use of growth rates in our subsequent simulation and prediction experiments.

\subsection{Simulation Experiments}

In order to evaluate the performance of the different models from the perspective of simulation, our four estimated models (RW, DS, SUROOT, and PAR) were used to simulate $N$ observations of $y_{t}$. The starting values for the simulations were fixed to be the last observation of the historical sample used in estimation. In this sense, following the theoretical arguments made in Corradi and Swanson (2011), simulated distributions for a given future period, say $h$, are asymptotically equivalent to $h$ step ahead predictive densities. Note that the models used for simulation are the models outlined above (see Section 2.1), including an error term, where the error is assumed to be iid normal, and where the variance of the error term is estimated using the residuals of the fitted model. Thus, simulation models take forms such as $\triangle_{12} y_{t}=1.66+\varepsilon_{t}$, for example, where $\varepsilon_{t} \sim i i d N\left(0, \sigma_{\varepsilon}^{2}\right)$, and $\sigma_{\varepsilon}^{2}$ is calibrated from the data. Results are also tabulated for the case where errors are drawn randomly from the empirical distribution of the residuals. Findings in this case are qualitatively similar to those reported below, and hence have been omitted for the sake of brevity (complete results are available upon request). We consider simulation paths of length $N=\{5 T, 10 T\}$, where $T$ is the historical sample size. All results are gathered in Table 3, and are based on the analysis of 100 simulation paths using the distribution comparison test discussed in Section 2.2 (further discussion of the experimental setup is contained in the footnote to Table 3; and a substantially more extensive simulation analysis is available upon request).

Our results based on the simulation experiments can be summarized as follows. First, the null hypothesis that there is nothing to choose between the different models based on the application

of the $Z_{T, S}$ test is not rejected for any variables, when the benchmark model is RW (compare the first column of entries in the table with the critical values reported in the $2^{\text {nd }}$ through $5^{\text {th }}$ columns 
of entries). However, this result may be in part be driven by poor finite sample test performance, as evidenced by the fact that there appear to be stark differences between the CS distributional loss measures reported in the last four columns of the table (see Section 2.2 for the definition of this MSFE type distributional loss measure). In particular, notice that for CPI1, PAR and RW CS distributional loss values are around one third as large as those associated with the DS model and one half as large as those associated with SUROOT. Overall, the PAR CS distributional loss is lowest for 9 of 14 variables, and is either lowest or very close to lowest for 14 of 14 variables. In contrast, the SUROOT model is lowest or very close to lowest for 0 of 14 variables. Results for the other two models are more mixed, with both RW and DS performing well for 7 of 14 variables. However, it should be stressed that we have: (i) set $y_{t}^{*}=\left(\Delta \ln X_{t}, \Delta \ln X_{t-1}\right.$ ); and (ii) set $U$ to be the entire range of the historical data. For different choice of $y_{t}^{*}$ and $U$, results may vary. For example, choosing $U$ to correspond to the tail of the distribution, or choosing $\phi(u)$ to weight "more important" regions of the distribution may yield different results. Nevertheless, based upon our somewhat naive application of the CS test, there appears to be substantive evidence in favor of the more general PAR model, particularly with respect to our simple SUROOT model. Of course, if the objective were to simulate $12^{\text {th }} \log$ differences, then the SUROOT model would be expected to perform much better (see Swanson and Urbach (2005) for evidence of this). This result underscores how important it is for the empiricist to carefully design an appropriate loss function based on her/his particular requirements, prior to selecting amongst alternative models. In particular, this finding underscores the importance of both choice of forecast or simulation horizon and choice between minimizing point or distribution based loss measures.

\subsection{Prediction Experiments}

The objective in the previous subsection was to assess the ability of the alternative models to simulate long paths of future observations. Clearly, each simulation path can be thought of as containing predictions for many different horizons, some of which are very far in the future. While this approach to model assessment has obvious uses in a variety of contexts, it is quite different from an assessment of the models based on their ability to predict a variable for a single given horizon.

In this subsection we report the results of prediction experiments for fixed forecast horizons. ${ }^{5}$

\footnotetext{
${ }^{5}$ For further discussion of the predictive performance of the 4 models analyzed in this paper (see Osborn, Heravi,
} 
Prediction models are constructed recursively, starting with $R=120-h+1$ observations, and ending with $R=T-h$ observations, where $T$ is the sample size, and $h$ is the forecast horizon (recall that $h$ is set equal to $1,3,12$, and 60 months). The results of these experiments are contained in Table 4, where MSFEs (Panel A) and DM predictive accuracy test statistics based on MSFE loss (Panel B) are reported. All DM tests are pairwise, and compare the benchmark RW model with the model denoted in the column header to the table. Negative values for DM statistics indicate that the point MSFE associated with the benchmark model is lower than that for the other model. Starred DM test statistics indicate rejection of the predictive accuracy null using $5 \%$ nominal size critical values (see Section 2.2 for further details).

Our tabulated results are consistent with the following conclusions. First, The SUROOT model performs admirably well for predicting one-step ahead (see Panel A of the table). Indeed, the SUROOT model dominates the RW model for 8 of 14 series, based on application of the DM predictive accuracy test (see Panel B of the table), and for 10 of 14 series based on point MSFE comparison. This is a somewhat surprising finding, given the evidence of the HEGY tests that there is little seasonal integration; but is completely consistent with the finding of Osborn et al. (1999) that seasonal difference models perform well when used to predict 2-digit industrial production series at short horizons, even though they find very little test-based evidence of seasonal integration. Thus, we again have evidence of the lack of reliability of in-sample integration tests.

Second, the results for $h=3,12$, and 60 are very different from those for $h=1$. The SUROOT model is never dominant based on accuracy tests, and is only point MSFE dominant for one variable at one horizon. Clearly, parameter estimation error plays a crucial role in empirical models of the variety examined here. Namely, even if one assumes that there is seasonal integration, in accord with our finding that SUROOT is dominant for $h=1$, PEE ensures that SUROOT is soundly beaten at all higher horizons. At the very least, we must conclude that SUROOT is a poor approximation when used to predict horizons greater than $h=1 .^{6}$

Third, The PAR model is reasonably accurate. In particular, PAR is point MSFE and DM test dominant for 8 of 14 variables, relative to the RW benchmark, for $h=1$. Furthermore, PAR and Birchenhall (1999) and Paap, Franses, and Hoek (1997)).

${ }^{6}$ It should be noted that, as we have mentioned, parameter estimation error is one key reason for the poor performance of the SUROOT model at higher forecast horizons. The evaluation of techniques that incorporate this information into potentially "improved" SUROOT models is left to future research. 
performs approximately as well as the RW model for 4 of the remaining 6 variables. Even more importantly, PAR continues to dominate RW, and indeed all other model, with respect to the same set of 8 variables across all forecast horizons. Overall, the set of variables for which PAR dominates include all variables other than our price and IP sub-component variables. This favorable evidence in support of the PAR model mimics the evidence presented in the previous subsection, where the PAR model was found to be the best performing model, based on CS distributional loss.

\section{Concluding Remarks}

We have provided evidence that a simple version of the seasonal unit root (SUROOT) model performs very well for predicting various economic variables when the forecast horizon is 1-step ahead. This result suggests that seasonal integration tests that reject the null of SUROOT need to be interpreted with caution. Their poor finite sample properties may mislead investigators into believing that seasonal unit root models are not useful (see Osborn et al. (1999) for a related discussion). However, for horizons of greater than one-step ahead, the SUROOT model performs poorly when used for prediction, suggesting that parameter estimation error is crucial to understanding the empirical performance of such models. This result is confirmed via a series of Monte Carlo experiments examining parameter estimation error, and via a series of experiments wherein (future) conditional distributions are simulated. Interestingly, simple periodic autoregressions do not have this property, and indeed perform very well in both prediction and simulation experiments, at all horizons considered in this paper. Deterministic seasonality models also perform reasonably well at all forecast horizons, and indeed dominate PAR models for a small subset of our variables, including price and IP sub-component variables. Finally, by comparing simulation and prediction based evidence, we underscore the importance of carefully designing on a case by case basis the criteria with which one judges alternative models. In particular, we underscore the importance of both choice of forecast or simulation horizon and choice between minimizing point or distribution based loss measures. 


\section{References}

Andrews, D.W.K., (1997), A Conditional Kolmogorov Test, Econometrica, 65, 1097-1128.

Beaulieu, J.J. and J. Miron, (1993), Seasonal Unit Roots and Deterministic Seasonals in Aggregate U.S. Data, Journal of Econometrics, 55, 305-328.

Bell, W.R., (1987), A Note on Overdifferencing and the Equivalence of Seasonal Time Series Models with Monthly Mean and Models with $(0,1,1)_{12}$ Seasonal Parts When $\theta=1$, Journal of Business and Economic Statistics, 5, 383-387.

Boswijk, H.P. and P.H. Franses, (1996), Unit Roots in Periodic Autoregressions, Journal of Time Series Analysis, 17, 221-245.

Cheng, Che-Hui, and P.-C. Wu (2013), Nonlinear Earnings Persistence, International Review of Economics and Finance, 25, 156-168.

Clark, T.E., and M.W., McCracken (2005), Evaluating Direct Multi-Step Forecasts, Econometric Reviews, 24, 369-404.

Corradi, V. and N.R. Swanson, (2002), A Consistent Test for Out of Sample Nonlinear Predictive Ability, Journal of Econometrics, 110, 353-381.

Corradi, V. and N.R. Swanson, (2006a), Predictive Density Evaluation, in: Handbook of Economic Forecasting, eds. Clive W.J. Granger, Graham Elliot and Allan Timmermann, Elsevier, Amsterdam, pp. 197-284.

Corradi, V. and N.R. Swanson, (2006b), Predictive Density and Conditional Confidence Intervals Accuracy Tests, Journal of Econometrics, 135, 187-228.

Corradi, Valentina and Norman R. Swanson, (2007a), Evaluation of Dynamic Stochastic General Equilibrium Models Based on Distributional Comparison of Simulated and Historical Data, Journal of Econometrics, 136, 699-723.

Corradi, Valentina and Norman R. Swanson, (2007b), Nonparametric Bootstrap Procedures for Predictive Inference Based on Recursive Estimation Schemes, International Economic Review, 48, 67-109.

Corradi, Valentina and Norman R. Swanson, (2011), Predictive Density Construction and Accuracy Testing with Multiple Possibly Misspecified Diffusion Models, Journal of Econometrics, 161, 2011, 304-324.

Diebold, F.X., and R.S. Mariano, (1995), Comparing Predictive Accuracy, Journal of Business and Economic Statistics, 13, 253-263.

Franses, P.H., (1996a), Periodicity and Stochastic Trends in Economic Time Series, Oxford, Oxford University Press.

Franses, P.H., (1996b), Recent Advances in Modelling Seasonality, Journal of Economic Surveys, 10, 299-345.

Franses, P.H., (2001), Some Comments on Seasonal Adjustment, Revista de Economia del Rosaro, 4, 9-16.

Franses, P.H., H. Hoek, and R. Paap, (1997), Bayesian Analysis of Seasonal Unit Roots and Seasonal Mean Shifts, Journal of Econometrics, 78, 359-380.

Franses, P.H. and R. Paap, (2002), Forecasting with Periodic Autoregressive Time Series Models, in: A Companion to Economic Forecasting, eds. M.P. Clements and D.F. Hendry, Oxford, Blackwell Publishers, pp. 432-452.

Franses, P.H. and D. van Dijk, (2005), The Forecasting Performance of Various Models for Seasonality and Nonlinearity for Quarterly Industrial Production, International Journal of Forecasting, 21, 87-102. 
Franses, P.H. and T. Vogelsang, (1998), On Seasonal Cycles, Unit Roots, and Mean Shifts, Review of Economics and Statistics, 80, 231-240.

Ghysels E. and D.R. Osborn, (2001), The Econometric Analysis of Seasonal Time Series, Cambridge, MA, Cambridge University Press.

Ghysels E., H.S. Lee and J. Noh, (1994), Testing for Unit Roots in Seasonal Time Series - Some Theoretical Extensions and A Monte Carlo Investigation, Journal of Econometrics, 62, 415-442.

Granger C.W.J. and T. Teräsvirta, (1993), Modelling Nonlinear Economic Relationships, Oxford, Oxford University Press.

Hamilton, J.D., (1994), Time Series Analysis, Princeton, Princeton University Press.

Hylleberg, S., (1992), Modelling Seasonality, Oxford, Oxford University Press.

Hylleberg, S., (1994), Modelling Seasonal Variation, in: Nonstationary Time Series Analysis and Cointegration, eds. C.P. Hargreaves, Oxford, Oxford University Press.

Hylleberg, S., R.F. Engle, C.W.J. Granger and B.S. Yoo, (1990), Seasonal Integration and Cointegration, Journal of Econometrics, 44, 215-238.

Kilian, L., (1999), Exchange Rates and Monetary Fundamentals: What Do We Learn from LongHorizon Regressions?, Journal of Applied Econometrics, 14, 491-510.

McCracken. M.W. and S. Sapp, (2004), Evaluating the Predictive Ability of Exchange Rates Using Long Horizon Regressions: Mind your p's and q's. Journal of Money, Credit and Banking, forthcoming.

Miron, J.A., (1996), The Economics of Seasonal Cycles, Cambridge, MA, MIT Press.

Miron, J.A. and J.J. Beaulieu, (1996), What Have Macroeconomists Learned About Business Cycles from the Study of Seasonal Cycles, Review of Economics and Statistics, 78, 54-66.

Osborn, D.R., (2002), Unit Roots versus Deterministic Representations of Seasonality for Forecasting, in: A Companion to Economic Forecasting, eds. M.P. Clements and D.F. Hendry, Oxford, Blackwell Publishers, pp. 409-431.

Osborn, D.R., S. Heravi, and C.R. Birchenhall, (1999), Seasonal Unit Roots and Forecasts of Two-Digit European Industrial Production, International Journal of Forecasting, 15, 27-47.

Osborn, D.R. and P.M.M. Rodrigues, (2002), Asymptotic Distributions of Seasonal Unit Root Tests: A Unifying Approach, Econometric Reviews, 21, 221-241.

Paap, R., P.H. Franses, and H. Hoek, (1997), Mean Shifts, Unit Roots, and Forecasting Seasonal Time Series, International Journal of Forecasting, 13, 357-36

Psaradakis, Z., (1997), Testing for Unit Roots in Time Series with Nearly Deterministic Seasonal Variation, Econometric Reviews, 16, 421-439.

Rodrigues, P.M.M., (2001), Near Seasonal Integration, Econometric Theory, 17, 70-86.

Rodrigues, P.M.M. and D.R. Osborn, (1999), Performance of Seasonal Unit Root Tests for Monthly Data, Journal of Applied Statistics, 26, 985-1004.

Swanson, N.R. and R. Urbach, (2013), Appendix: Prediction and Simulation Using Simple Models Characterized by Nonstationarity and Seasonality, Working Paper, Rutgers University.

Urga, Giovani and P. Aroca-Gonzalez, (2000), Hausman Tests for Seasonal Unit Roots in Presence of MA(1) Errors, mimeo, Cass Business School, City University of London.

West, K., (1996), Asymptotic Inference About Predictive Ability, Econometrica, 64, 1067-1084.

Zhu, M., (2013), Return distribution predictability and its implications for portfolio selection, International Review of Economics and Finance, 27, 209-223. 
Figure 1: Simulated CPI Levels and 1st Log Differences Simulation Models Calibrated Using Monthly U.S. Data
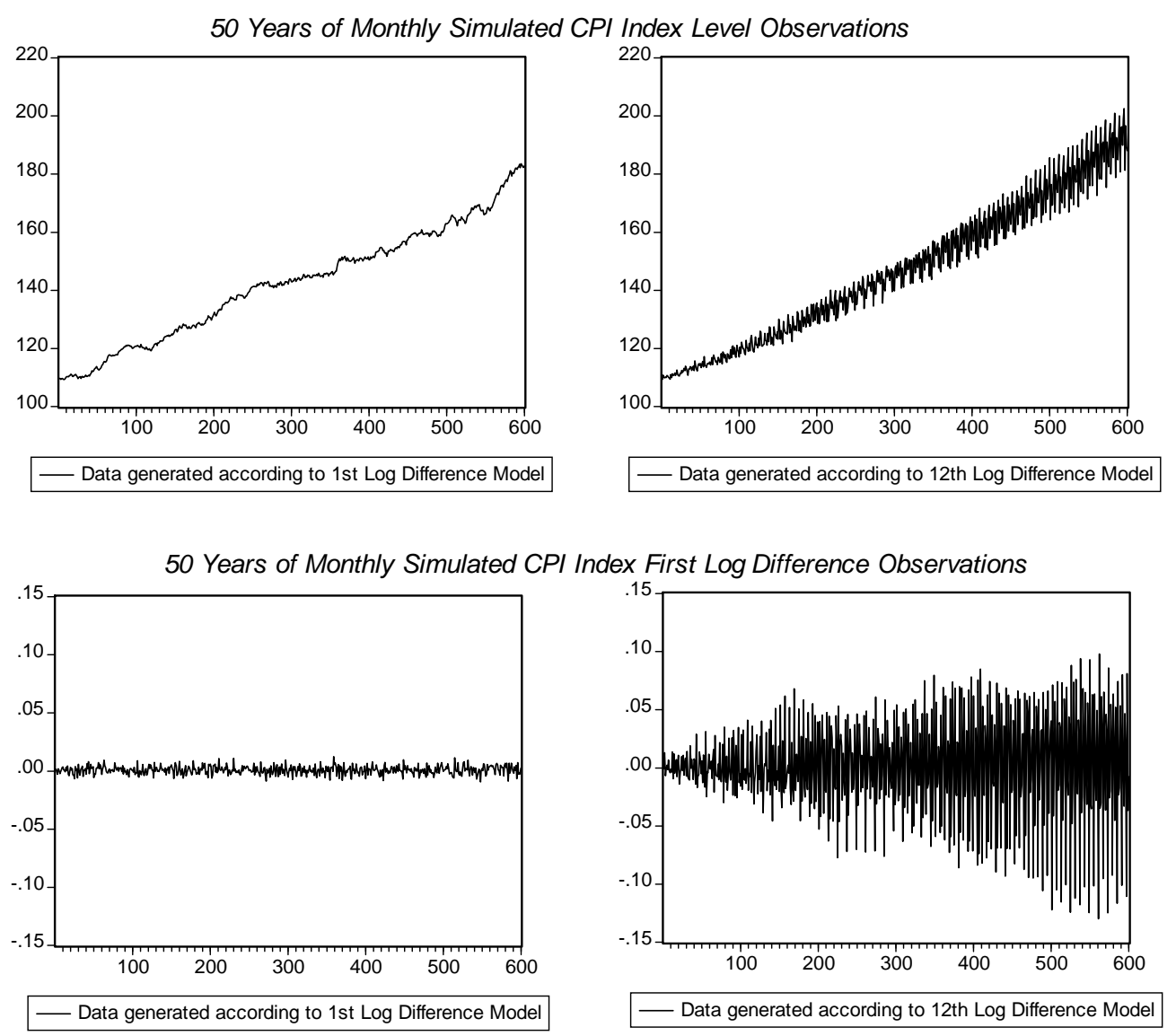
Figure 2: Macroeconomic Variables - Growth Rates for the Period 1959:1-2005:12

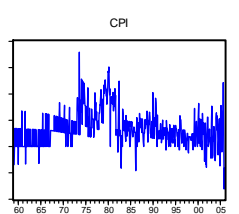

Housing Starts

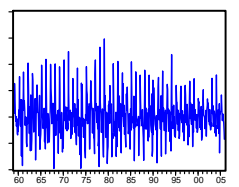

IP-durable consumergoods

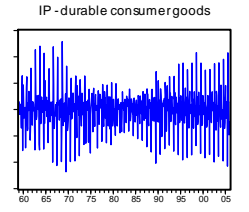

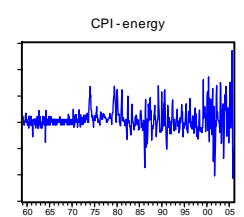

Industrial Production (IP)

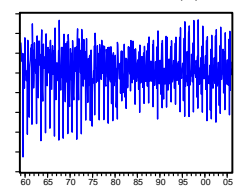

IP-nondurable consumergoods

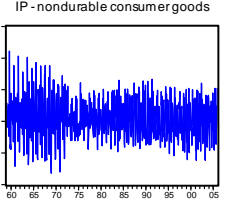

Total inventonies-mantacturing

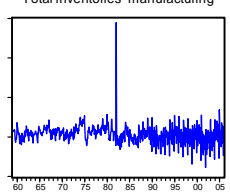

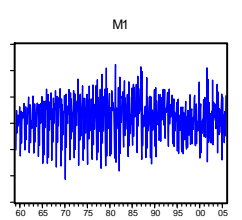

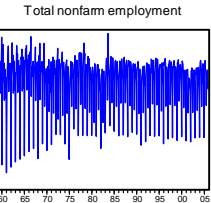

Motorvehicle unitretail sales

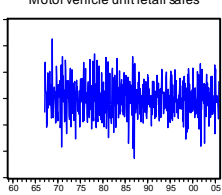

Retail sales

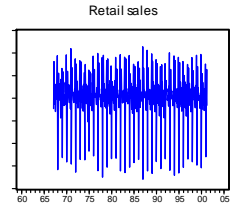

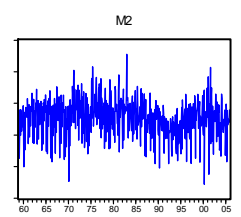
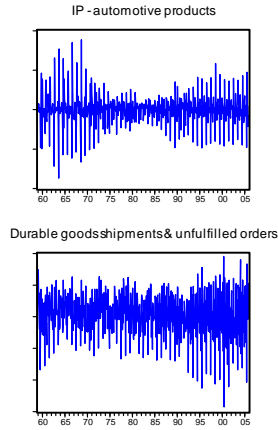

Figure 3: Macroeconomic Variables - Estimated Spectra
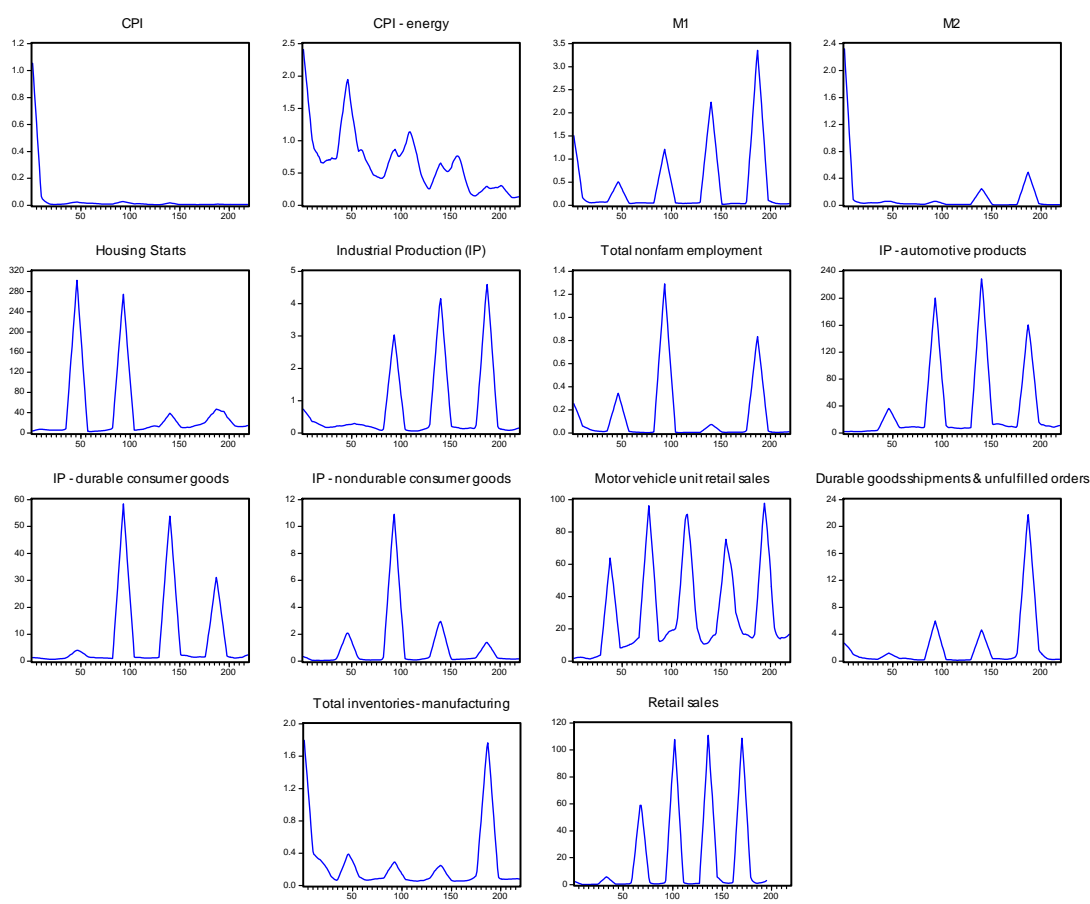
Table 1: Seasonality Evidence - Diagnostic Statistics for Macroeconomic Variables $\left.{ }^{*}\right)$

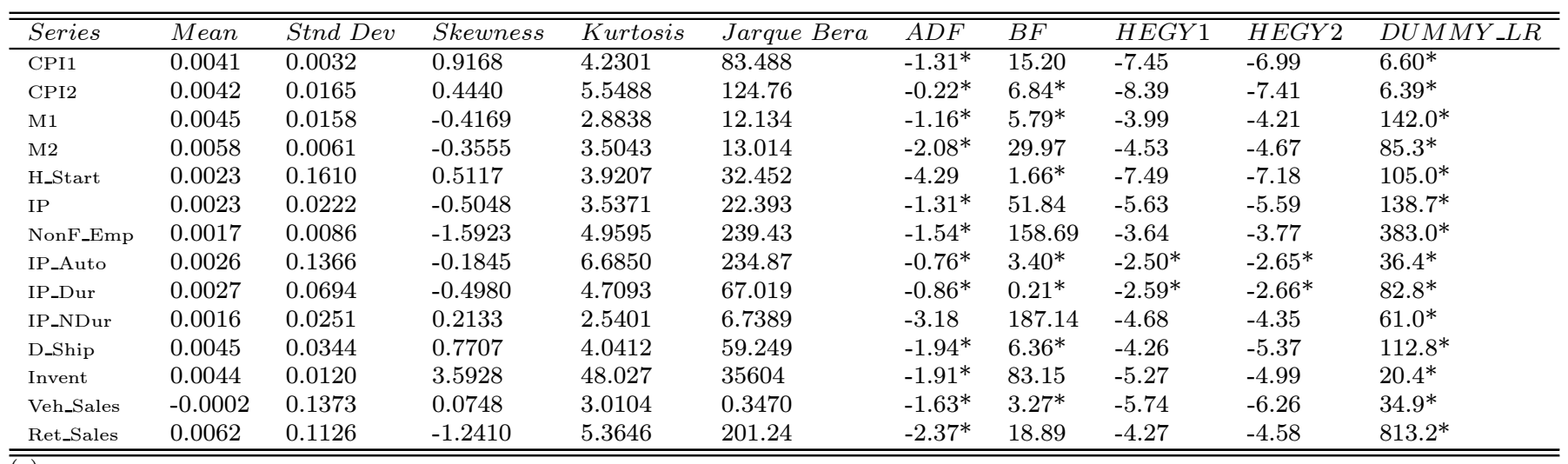

(*) Various summary measures for the 14 series examined in the paper are contained in this table. The series consist of: two money stock variables (M1 and M2); two CPI series including CPI for all urban consumers, all items (CPI1), and energy (CPI2); housing starts (H_Start); industrial production (IP); total nonfarm employment (NonF_Emp); industrial production - automotive products (IP_Auto); industrial production - durable consumer goods (IP_Dur); industrial production - durable consumer goods (IP_NDur); durable goods shipments of new orders and unfilled orders (D_Ship); total inventories, manufacturing (Invent); retail sales (Ret_sales) - for the period 1967:1-2001:4; and motor vehicle unit retail sales (Veh_sales) - for the period 1967:1-2005:12. Data are monthly U.S. figures for the period 1959:1-2005:12, except where noted. Mean, Stnd Dev, Skewness, Kurtosis, Jarque-Bera, and Dummy_LM statistics are calculated using log differenced data, while unit root tests are carried out using logged data. For the unit root tests, starred entries represent cases where the null of unit root fails to reject at a $5 \%$ nominal level. HEGY test statistics are reported for seasonal frequency $\pi_{2}$ (see above for further discussion), and starred entries denote a failure to reject the seasonal unit root null at a $5 \%$ level. The last column reports likelihood ratio statistics for testing the null hypothesis that $\theta_{s}=\theta, s=1 \cdots 12$ in the standard deterministic dummay variable model (i.e. see model DS above). Starred entries indicate rejection of the null at 5\% level. Further details are contained in Section 2. 
Table 2: Monte Carlo Results: MSFE Comparison, DM Predictive Accuracy Analysis, and

$$
\text { Seasonality Test Performance }{ }^{(*)}
$$

\begin{tabular}{|c|c|c|c|c|c|c|c|c|c|c|c|c|c|c|c|}
\hline \multirow{3}{*}{$\begin{array}{c}D G P \\
\left(\theta, \sigma_{\varepsilon}, \phi\right)\end{array}$} & \multicolumn{4}{|c|}{ Point MSFE comparison } & \multicolumn{8}{|c|}{$\overline{D M}$ Test Win Frequency } & \multicolumn{3}{|c|}{ Seasonality Test Size } \\
\hline & \multirow[t]{2}{*}{$h=1$} & \multirow[t]{2}{*}{$h=3$} & \multirow[t]{2}{*}{$h=12$} & \multirow[t]{2}{*}{$h=60$} & \multicolumn{2}{|c|}{$h=1$} & \multicolumn{2}{|c|}{$h=3$} & \multicolumn{2}{|c|}{$h=12$} & \multicolumn{2}{|c|}{$h=60$} & \multirow[t]{2}{*}{$B F$} & \multirow[t]{2}{*}{$H E G Y 1$} & \multirow[t]{2}{*}{$H E G Y 2$} \\
\hline & & & & & $S R T$ & $R W$ & $S R T$ & $R W$ & $S R T$ & $R W$ & $S R T$ & $R W$ & & & \\
\hline$(-0.6,0.005,0.0)$ & 1.00 & 1.00 & 0.87 & 0.67 & 1.00 & 0.00 & 1.00 & $\overline{0.00}$ & 0.30 & 0.00 & 0.07 & 0.05 & 0.64 & 0.46 & 0.45 \\
\hline$(-0.9,0.005,0.0)$ & 1.00 & 1.00 & 0.70 & 0.20 & 1.00 & 0.00 & 0.97 & 0.00 & 0.11 & 0.05 & 0.02 & 0.27 & 0.34 & 1.00 & 1.00 \\
\hline$(-0.6,0.01,0.0)$ & 1.00 & 1.00 & 0.90 & 0.80 & 1.00 & 0.00 & 1.00 & 0.00 & 0.32 & 0.00 & 0.10 & 0.05 & 0.61 & 0.38 & 0.36 \\
\hline$(-0.9,0.01,0.0)$ & 1.00 & 0.97 & 0.70 & 0.30 & 0.97 & 0.00 & 0.95 & 0.00 & 0.14 & 0.11 & 0.04 & 0.19 & 0.34 & 1.00 & 1.00 \\
\hline$(-0.6,0.1,0.0)$ & 1.00 & 1.00 & 0.90 & 0.87 & 1.00 & 0.00 & 1.00 & 0.00 & 0.35 & 0.01 & 0.17 & 0.04 & 0.54 & 0.41 & 0.39 \\
\hline$(-0.9,0.1,0.0)$ & 1.00 & 0.95 & 0.75 & 0.25 & 1.00 & 0.00 & 0.94 & 0.00 & 0.15 & 0.07 & 0.03 & 0.26 & 0.30 & 1.00 & 1.00 \\
\hline$(-0.6,0.005,0.5)$ & 1.00 & 1.00 & 0.86 & 0.76 & 1.00 & 0.00 & 1.00 & 0.00 & 0.26 & 0.01 & 0.17 & 0.07 & 0.58 & 0.34 & 0.33 \\
\hline$(-0.9,0.005,0.5)$ & 1.00 & 0.97 & 0.57 & 0.27 & 1.00 & 0.00 & 0.96 & 0.00 & 0.17 & 0.12 & 0.02 & 0.32 & 0.35 & 1.00 & 1.00 \\
\hline$(-0.6,0.01,0.5)$ & 1.00 & 1.00 & 0.82 & 0.71 & 1.00 & 0.00 & 1.00 & 0.00 & 0.31 & 0.02 & 0.11 & 0.10 & 0.62 & 0.38 & 0.36 \\
\hline$(-0.9,0.01,0.5)$ & 1.00 & 0.98 & 0.62 & 0.22 & 1.00 & 0.00 & 0.98 & 0.00 & 0.22 & 0.12 & 0.02 & 0.26 & 0.34 & 1.00 & 1.00 \\
\hline$(-0.6,0.1,0.5)$ & 1.00 & 1.00 & 0.90 & 0.84 & 1.00 & 0.00 & 1.00 & 0.00 & 0.28 & 0.00 & 0.14 & 0.02 & 0.58 & 0.42 & 0.43 \\
\hline$(-0.9,0.1,0.5)$ & 1.00 & 0.99 & 0.50 & 0.21 & 1.00 & 0.00 & 0.97 & 0.00 & 0.10 & 0.18 & 0.02 & 0.25 & 0.33 & 1.00 & 1.00 \\
\hline$(-0.6,0.005,-0.5)$ & 1.00 & 1.00 & 0.86 & 0.76 & 0.95 & 0.00 & 1.00 & 0.00 & 0.24 & 0.01 & 0.16 & 0.06 & 0.62 & 0.32 & 0.35 \\
\hline$(-0.9,0.005,-0.5)$ & 1.00 & 0.96 & 0.66 & 0.36 & 1.00 & 0.00 & 0.95 & 0.00 & 0.16 & 0.12 & 0.06 & 0.31 & 0.41 & 1.00 & 1.00 \\
\hline$(-0.6,0.01,-0.5)$ & 1.00 & 1.00 & 0.94 & 0.74 & 1.00 & 0.00 & 1.00 & 0.00 & 0.34 & 0.01 & 0.14 & 0.03 & 0.60 & 0.37 & 0.39 \\
\hline$(-0.9,0.01,-0.5)$ & 1.00 & 0.97 & 0.67 & 0.19 & 1.00 & 0.00 & 0.96 & 0.00 & 0.11 & 0.12 & 0.02 & 0.32 & 0.33 & 1.00 & 1.00 \\
\hline$(-0.6,0.1,-0.5)$ & 1.00 & 1.00 & 0.84 & 0.68 & 1.00 & 0.00 & 1.00 & 0.00 & 0.24 & 0.01 & 0.18 & 0.04 & 0.57 & 0.34 & 0.37 \\
\hline$(-0.9,0.1,-0.5)$ & 1.00 & 0.96 & 0.49 & 0.17 & 1.00 & 0.00 & 0.95 & 0.01 & 0.09 & 0.11 & 0.07 & 0.24 & 0.30 & 1.00 & 1.00 \\
\hline
\end{tabular}

${ }^{(*)}$ Results based upon a (i) series of prediction experiments, and (ii) in-sample HEGY testings are reported in this table. With regard to the prediction experiments, in each simulation a sample of 500 observations was drawn, and prediction models were recursively estimated, begininng with an in-sample period of $R=120-h+1$ observations, and yielding a sequence of 380 ex-ante $h$-step ahead forecasts. Results reported in the first 12 columns of numerical entries in the table are based upon examination of these forecasts. Namely, the first 4 columns report the proportion of simulations for which the SUROOT model predictions were "MSFE-better" than those based on the $R W$ model. The next 8 columns report the proportion of simulations for which the two models yielded "MSFE-better" predictions based upon application of the Diebold-Mariano (1995) predictive accuracy test at a 5\% nominal significance level, based on MSFE loss (see Section 4 for further details). Entires in the last three columns of the table indicate the proportion of times that the seasonal unit root (HEGY) and BF tests reject the null (see Table 1 and Section 2 for further details). All results are based on 500 Monte Carlo simulations. 
Table 3: Distributional Accuracy Tests Based on the Comparison of Historical and Simulated Data - Benchmark Model is RW $\left.{ }^{*}\right)$

\begin{tabular}{|c|c|c|c|c|c|c|c|c|c|c|}
\hline \multirow[t]{2}{*}{ Series } & \multirow[t]{2}{*}{$S, l$} & \multirow[t]{2}{*}{$Z$} & \multicolumn{2}{|c|}{ Crit Val $\left(Z^{*}\right)$} & \multicolumn{2}{|c|}{ Crit Val $\left(Z^{* *}\right)$} & \multicolumn{4}{|c|}{ CS Distributional Loss } \\
\hline & & & $10 \%$ & $5 \%$ & $10 \%$ & $5 \%$ & $R W$ & $D S$ & SUROOT & $P A R$ \\
\hline \multirow[t]{2}{*}{ CPI1 } & $5 \mathrm{~T}, 4$ & -0.0031 & 2.893 & 2.9863 & 2.8997 & 2.9954 & 1.5634 & 4.7753 & 2.5993 & 1.5665 \\
\hline & $10 \mathrm{~T}, 12$ & -0.0048 & 2.6184 & 2.8337 & 2.6068 & 2.8256 & 1.5631 & 4.9371 & 2.6047 & 1.5679 \\
\hline \multirow[t]{2}{*}{ CPI2 } & $5 \mathrm{~T}, 4$ & -0.0067 & 0.2552 & 0.2652 & 0.2518 & 0.2673 & 1.1283 & 1.1412 & 1.3952 & 1.1351 \\
\hline & $10 \mathrm{~T}, 12$ & -0.0022 & 0.2602 & 0.2676 & 0.2613 & 0.2710 & 1.1285 & 1.1446 & 1.3959 & 1.1307 \\
\hline \multirow[t]{2}{*}{ M1 } & $5 \mathrm{~T}, 4$ & 0.0174 & 0.6724 & 0.704 & 0.6696 & 0.6990 & 2.2174 & 2.2000 & 2.9743 & 2.2013 \\
\hline & $10 \mathrm{~T}, 12$ & 0.0167 & 0.6774 & 0.7037 & 0.6729 & 0.7040 & 2.2167 & 2.2000 & 2.9743 & 2.2005 \\
\hline \multirow[t]{2}{*}{ M2 } & $5 \mathrm{~T}, 4$ & 0.0046 & 0.8092 & 0.9512 & 0.8069 & 0.9393 & 1.8239 & 2.4718 & 3.1176 & 1.8193 \\
\hline & $10 \mathrm{~T}, 12$ & 0.0042 & 0.9044 & 1.0065 & 0.9025 & 1.0039 & 1.8234 & 2.4716 & 3.157 & 1.8192 \\
\hline \multirow[t]{2}{*}{ H_Start } & $5 \mathrm{~T}, 4$ & 0.0077 & 0.0627 & 0.0796 & 0.0658 & 0.0814 & 1.9628 & 1.9551 & 2.0481 & 1.9570 \\
\hline & $10 \mathrm{~T}, 12$ & 0.0104 & 0.0784 & 0.0859 & 0.0789 & 0.0877 & 1.9650 & 1.9546 & 2.0514 & 1.9575 \\
\hline \multirow[t]{2}{*}{$\overline{\mathrm{IP}}$} & $5 \mathrm{~T}, 4$ & 0.0151 & 0.3824 & 0.4107 & 0.3845 & 0.4119 & 2.0792 & 2.0677 & 2.5311 & 2.0641 \\
\hline & $10 \mathrm{~T}, 12$ & 0.0167 & 0.4067 & 0.4172 & 0.4059 & 0.4183 & 2.0808 & 2.0667 & 2.5327 & 2.0641 \\
\hline \multirow[t]{2}{*}{ NonF_Emp } & $5 \mathrm{~T}, 4$ & 0.1401 & 0.6085 & 0.6974 & 0.5943 & 0.6931 & 2.0522 & 1.9366 & 2.8813 & 1.9121 \\
\hline & $10 \mathrm{~T}, 12$ & 0.1237 & 0.6524 & 0.7127 & 0.6629 & 0.7195 & 2.0363 & 1.9397 & 2.8811 & 1.9126 \\
\hline \multirow[t]{2}{*}{ IP_Auto } & $5 \mathrm{~T}, 4$ & 0.0231 & 0.1317 & 0.1372 & 0.1317 & 0.1358 & 1.3325 & 1.3291 & 1.4475 & 1.3094 \\
\hline & $10 \mathrm{~T}, 12$ & 0.0198 & 0.11 & 0.1167 & 0.1098 & 0.1153 & 1.3288 & 1.3299 & 1.4159 & 1.309 \\
\hline \multirow[t]{2}{*}{ IP_Dur } & $5 \mathrm{~T}, 4$ & 0.0217 & 0.2924 & 0.3102 & 0.2938 & 0.3075 & 1.8412 & 1.8301 & 2.1449 & 1.8195 \\
\hline & $10 \mathrm{~T}, 12$ & 0.0219 & 0.3205 & 0.3381 & 0.3201 & 0.3388 & 1.8421 & 1.8307 & 2.1624 & 1.8202 \\
\hline \multirow[t]{2}{*}{ IP_NDur } & $5 \mathrm{~T}, 4$ & 0.0015 & 0.3974 & 0.4088 & 0.4014 & 0.407 & 2.2186 & 2.2929 & 2.6528 & 2.2171 \\
\hline & $10 \mathrm{~T}, 12$ & 0.0018 & 0.3969 & 0.4051 & 0.3967 & 0.4062 & 2.2195 & 2.2971 & 2.6557 & 2.2177 \\
\hline \multirow[t]{2}{*}{ D_Ship } & $5 \mathrm{~T}, 4$ & 0.0058 & 0.2615 & 0.2807 & 0.2605 & 0.2777 & 1.8453 & 1.8419 & 2.1582 & 1.8395 \\
\hline & $10 \mathrm{~T}, 12$ & 0.0082 & 0.2638 & 0.2783 & 0.2668 & 0.2782 & 1.8466 & 1.8408 & 2.1629 & 1.8384 \\
\hline \multirow[t]{2}{*}{$\begin{array}{l}\text { Invent } \\
\end{array}$} & $5 \mathrm{~T}, 4$ & -0.0178 & 0.5438 & 0.5506 & 0.5426 & 0.5497 & 0.7184 & 0.8054 & 1.2663 & 0.7362 \\
\hline & $10 \mathrm{~T}, 12$ & -0.0186 & 0.5291 & 0.5354 & 0.5292 & 0.5354 & 0.7183 & 0.7976 & 1.2663 & 0.7369 \\
\hline \multirow[t]{2}{*}{ Ret_Sales } & $5 \mathrm{~T}, 4$ & 0.1019 & 0.3401 & 0.3461 & 0.3411 & 0.353 & 1.9304 & 2.0016 & 2.2441 & 1.8285 \\
\hline & $10 \mathrm{~T}, 12$ & 0.1004 & 0.3368 & 0.3507 & 0.3366 & 0.3502 & 1.9291 & 2.0002 & 2.2407 & 1.8286 \\
\hline \multirow[t]{2}{*}{ Veh_Sales } & $5 \mathrm{~T}, 4$ & 0.0011 & 0.0556 & 0.0627 & 0.0538 & 0.0623 & 1.7596 & 1.7616 & 1.8359 & 1.7585 \\
\hline & 10T,12 & 0.0006 & 0.0495 & 0.0553 & 0.0502 & 0.0553 & 1.7590 & 1.7606 & 1.8259 & 1.7584 \\
\hline
\end{tabular}

${ }^{(*)} Z_{T, S}$ test statistics (called $Z$ in the table), and associated distributional loss measures (denoted CS Distributional Loss) are reported, where $S$ denotes the length of the simulated data series used in test statistic construction, and $T$ is the historical sample length, assumed to be the entire historical sample period (see footnote to Table 1). Note that this historical period is also the period used to estimate the models. As usual, the models are denoted by $R W, D S, S U R O O T$, and $P A R$ (see Section 2 for further details). The $Z_{T, S}$ test is designed to facillitate selection amongst alternative simulation models via comparison of simulated and historical distribtutions In the test, the benchmark model is $R W$, against which all other model are compared. The null hypothesis corresponds to the case where no alternative model outperforms the benchmark. Critical values that are constructed both assuming that $T / S$ approaches $\gamma>0\left(Z^{* *}\right)$ and assuming that $T / S$ approaches $0\left(Z^{*}\right)$, as $T$ and $S$ increase are reported in the 3th through 6th columns of entries (see Section 2 and Corradi and Swanson (2007a) for complete details). Further, $l$ is the bootstrap block length, and all statistics are based on a grid of 20x20 values for $u$, distributed uniformly across the historical data range. Bootstrap empirical distributions are constructed using 100 bootstrap replications. 
Table 4: Predictive Accuracy Test Results for Various Macroeconomic Variables $\left.{ }^{*}\right)$

\begin{tabular}{|c|c|c|c|c|c|c|c|c|c|c|c|c|}
\hline \multirow[b]{2}{*}{ Series } & \multicolumn{3}{|c|}{$h=1$} & \multicolumn{3}{|c|}{$h=3$} & \multicolumn{3}{|c|}{$h=12$} & \multicolumn{3}{|c|}{$h=60$} \\
\hline & $D S$ & SUROOT & $P A R$ & $D S$ & SUROOT & $P A R$ & $D S$ & SUROOT & $P A R$ & $D S$ & SUROOT & $P A R$ \\
\hline \multicolumn{13}{|c|}{ Panel A: MSFE for DS, SUROOT and PAR, Relative to the RW Model } \\
\hline$\overline{\text { CPI1 }}$ & 1.24 & 1.17 & 1.35 & 0.98 & 2.41 & 1.27 & 0.99 & 2.35 & 1.25 & 0.98 & 1.74 & 1.10 \\
\hline CPI2 & 0.88 & 1.68 & 2.37 & 1.07 & 3.28 & 1.04 & 1.03 & 3.23 & 1.03 & 1.04 & 2.23 & 1.02 \\
\hline M1 & 1.19 & 0.53 & 0.57 & 1.03 & 3.28 & 0.45 & 2.38 & 3.73 & 0.45 & 2.34 & 2.36 & 0.45 \\
\hline M2 & 1.97 & 0.88 & 0.85 & 1.57 & 5.69 & 0.75 & 9.05 & 5.61 & 0.78 & 9.55 & 2.53 & 0.76 \\
\hline H_Start & 1.57 & 0.98 & 0.46 & 5.49 & 1.58 & 0.43 & 2.00 & 2.50 & 0.43 & 2.05 & 1.57 & 0.43 \\
\hline IP & 1.26 & 0.53 & 0.79 & 1.27 & 3.90 & 0.67 & 1.91 & 3.32 & 0.65 & 2.00 & 2.97 & 0.65 \\
\hline NonF_Emp & 9.69 & 0.14 & 0.55 & 1.09 & 3.77 & 0.36 & 1.37 & 3.53 & 0.36 & 1.47 & 2.07 & 0.37 \\
\hline IP_Auto & 0.98 & 0.39 & 2.94 & 1.05 & 1.66 & 3.65 & 1.06 & 2.13 & 3.67 & 1.10 & 2.38 & 3.67 \\
\hline IP_Dur & 0.94 & 0.38 & 1.55 & 1.01 & 2.24 & 1.42 & 1.45 & 2.50 & 1.45 & 1.41 & 2.24 & 1.45 \\
\hline IP_NDur & 2.20 & 0.56 & 2.5 & 1.37 & 2.19 & 2.06 & 1.58 & 2.58 & 2.11 & 1.65 & 2.15 & 2.11 \\
\hline D_Ship & 1.05 & 0.52 & 0.43 & 1.26 & 2.95 & 0.46 & 1.01 & 2.99 & 0.46 & 1.00 & 2.12 & 0.46 \\
\hline Invent & 2.38 & 1.65 & 1.18 & 1.2 & 3.86 & 0.85 & 1.28 & 4.21 & 0.95 & 1.43 & 2.48 & 0.94 \\
\hline Ret_Sales & 7.87 & 0.08 & 0.06 & 2.75 & 0.77 & 0.07 & 2.55 & 1.58 & 0.06 & 2.61 & 2.64 & 0.06 \\
\hline Veh_Sales & 1.07 & 1.67 & 1.55 & 2.85 & 2.36 & 1.20 & 1.47 & 2.49 & 1.23 & 1.63 & 2.39 & 1.23 \\
\hline \multicolumn{13}{|c|}{ Panel B: DM Predictive Accuracy Test Statistics - Benchmark is the RW Model } \\
\hline CPI1 & -1.51 & -1.36 & $-2.26^{*}$ & 0.77 & $-3.81^{*}$ & $-2.62^{*}$ & 0.33 & $-4.67^{*}$ & $-2.57^{*}$ & 0.85 & $-3.53^{*}$ & -1.64 \\
\hline CPI2 & $2.01^{*}$ & $-2.77^{*}$ & $-3.79^{*}$ & -1.82 & $-3.53^{*}$ & -1.11 & -1.55 & $-3.05^{*}$ & -0.97 & -1.48 & $-3.16^{*}$ & -0.62 \\
\hline M1 & $-4.2^{*}$ & $3.07^{*}$ & $5.29^{*}$ & $-3.85^{*}$ & $-5.23^{*}$ & $4.11^{*}$ & $-8.33^{*}$ & $-4.39^{*}$ & $4.07^{*}$ & $-8.22^{*}$ & $-4.46^{*}$ & $4.08^{*}$ \\
\hline M2 & $-7.51^{*}$ & 0.73 & 1.64 & $-6.01^{*}$ & $-5.19^{*}$ & $3.07^{*}$ & $-2.16^{*}$ & $-3.95^{*}$ & $2.68^{*}$ & -1.89 & $-4.32^{*}$ & $3.00^{*}$ \\
\hline H_Start & $-4.15^{*}$ & 0.37 & $5.8^{*}$ & $-5.26^{*}$ & $-2.81^{*}$ & $6.04^{*}$ & $-5.68^{*}$ & $-5.78^{*}$ & $6.04^{*}$ & $-5.75^{*}$ & $-3.75^{*}$ & $6.04^{*}$ \\
\hline IP & $-5.12^{*}$ & $3.87^{*}$ & $2.33^{*}$ & $-6.24^{*}$ & $-5.1^{*}$ & $3.42 *$ & $-7.95^{*}$ & $-4.82^{*}$ & $3.73^{*}$ & $-8.35^{*}$ & $-5.18^{*}$ & $3.73^{*}$ \\
\hline NonF_Emp & $-3.96^{*}$ & $7.37^{*}$ & $6.65^{*}$ & $-6.36^{*}$ & $-7.96^{*}$ & $6.88^{*}$ & $-6.16^{*}$ & $-5.14^{*}$ & $6.85^{*}$ & $-6.08^{*}$ & $-7.18^{*}$ & $6.82^{*}$ \\
\hline IP_Auto & 0.89 & $4.37^{*}$ & $-5.63^{*}$ & $-4.78^{*}$ & $-3.22^{*}$ & $-5.51^{*}$ & $-5.83^{*}$ & $-6.63^{*}$ & $-5.59^{*}$ & $-5.23^{*}$ & $-4.6^{*}$ & $-5.59^{*}$ \\
\hline IP_Dur & $2.29 *$ & $4.68^{*}$ & $-3.75^{*}$ & $-3.04^{*}$ & $-5.16^{*}$ & $-2.89^{*}$ & $-6.86^{*}$ & $-7.87^{*}$ & $-3.10^{*}$ & $-6.65^{*}$ & $-5.34^{*}$ & $-3.11^{*}$ \\
\hline IP_NDur & $-9.32^{*}$ & $6.09 *$ & $-4.81^{*}$ & $-4.99^{*}$ & $-4.02^{*}$ & $-4.63^{*}$ & $-4.45^{*}$ & $-4.79^{*}$ & $-4.78^{*}$ & $-6.03^{*}$ & $-5.34^{*}$ & $-4.79 *$ \\
\hline D_Ship & $-2.98^{*}$ & $4.45^{*}$ & $6.17^{*}$ & $-5.19^{*}$ & $-5.25^{*}$ & $6.82^{*}$ & -1.82 & $-8.5^{*}$ & $6.82^{*}$ & 0.00 & $-6.03^{*}$ & $6.82^{*}$ \\
\hline Invent & $-3.48^{*}$ & -0.89 & -1.83 & $-2.3^{*}$ & $-2.46^{*}$ & $2.48^{*}$ & $-2.09^{*}$ & $-1.98^{*}$ & 1.41 & $-2.09^{*}$ & $-2.25^{*}$ & 1.55 \\
\hline Ret_Sales & $-2.62 *$ & $4.52 *$ & $4.59^{*}$ & $-4.07 *$ & 1.95 & $4.6^{*}$ & $-8.13^{*}$ & $-5.29 *$ & $4.61^{*}$ & $-8.19^{*}$ & $-5.28 *$ & $4.61 *$ \\
\hline Veh_Sales & $-2.85^{*}$ & $-2.89^{*}$ & $-2.89^{*}$ & $-4.09^{*}$ & $-2.61^{*}$ & $-2.16^{*}$ & $-4.92^{*}$ & $-2.57^{*}$ & $-2.36^{*}$ & $-5.87^{*}$ & $-3.36^{*}$ & $-2.36^{*}$ \\
\hline
\end{tabular}

(*) MSFEs and DM predictive accuracy test statistics based on MSFE loss are reported in the two panels of this table. All DM tests are pairwise, and compare the benchmark $R W$ model with the model denoted in the column header to the table. Negative values for DM statistics indicate that the point MSFE associated with the benchmark model is lower than that for the other model. Starred DM test statistics indicate rejection of the predictive accuracy null using 5\% nominal size critical values (see Section 2 for further details). Prediction models are constructed recursively, starting with $R=120-h+1$ observations, and ending with $R=T-h$ observations, where $T$ is the sample size, and $h$ is the forecast horizon (set equal to $1,3,12$, and 60 months ahead). For variable definitions, refer to Table 1. 